\title{
Radio polarimetry of compact steep spectrum sources at sub-arcsecond resolution ${ }^{\star}$
}

\author{
F. Mantovani ${ }^{1,2}$, A. Rossetti ${ }^{1}$, W. Junor ${ }^{3}$, D. J. Saikia ${ }^{4,5}$, and C. J. Salter ${ }^{6}$ \\ ${ }^{1}$ Istituto di Radioastronomia - INAF, via Gobetti 101, 40129 Bologna, Italy \\ e-mail: fmantovani@ira.inaf.it \\ 2 Max-Planck-Institut für Radioastronomie, Auf dem Hügel 69, 53121 Bonn, Germany \\ 3 Los Alamos National Laboratory, Los Alamos, NM 87545, USA \\ ${ }^{4}$ Cotton College State University, Panbazar, 781001 Guwahati, India \\ 5 National Centre for Radio Astrophysics, TIFR, Post Bag 3, Ganeshkhind, 411007 Pune, India \\ ${ }^{6}$ Arecibo Observatory, HC3 Box 53995, 00612 Arecibo, Puerto Rico
}

Received 21 November 2012 / Accepted 26 April 2013

\begin{abstract}
Aims. We report new Very Large Array (VLA) polarimetric observations of compact steep-spectrum (CSS) sources at 8.4, 15, and $23 \mathrm{GHz}$.

Methods. Using multi-frequency VLA observations we have derived sub-arcsecond resolution images of the total intensity, polarisation, and rotation measure $(\mathrm{RM})$ distributions.

Results. We present multi-frequency VLA polarisation observations of CSS sources. About half of the sources are point-like even at the resolution of $\sim 0.1 \times 0.1 \mathrm{arcsec}$. The remaining sources have double or triple structure. Low values for the percentage of polarised emission in CSS sources is confirmed. On the average, quasars are more polarised than galaxies. A wide range of RM values have been measured. There are clear indications of very large RMs up to $\approx 5585 \mathrm{rad} \mathrm{m}^{-2}$. CSS galaxies are characterized by RM values that are larger than CSS quasars. The majority of the objects show very large values of RM.

Conclusions. The available data on sub-arcsecond-scale rest-frame RM estimates for CSS sources show that these have a wide range of values extending up to $\sim 36000 \mathrm{rad} \mathrm{m}^{-2}$. RM estimates indicate an overall density of the magneto-ionic medium larger than classical radio sources.
\end{abstract}

Key words. polarization - quasars: general - galaxies: jets - radio continuum: galaxies

\section{Introduction}

The number of compact steep-spectrum (CSS) sources with detailed polarimetric information available at sub-arcsecond resolution is still small. We have conducted a series of polarimetric observations of CSS sources using the Very Large Array (VLA).

CSS objects are young radio sources, with ages $<10^{3-5} \mathrm{yr}$ (Fanti et al. 1990). They have linear sizes $\leq 20 \mathrm{kpc}^{1}$ and steep high-frequency radio spectra $\left(\alpha>0.5 ; S_{v} \propto v^{-\alpha}\right)$. Being subgalactic in size, CSS sources reside within their host galaxies. Therefore, Faraday rotation effects are to be expected when their polarised synchrotron emission is observed through the magneto-ionic interstellar medium (ISM) of the host galaxy. The comparison of polarised emission over a range of wavelengths is an important diagnostic of the physical conditions within and around these compact radio sources.

Existing sub-arcsec polarimetry has provided evidence in favour of the interaction of components of CSSs with dense gas clouds, (for example, see Junor et al. 1999).

Aiming at a deeper understanding of the CSS source phenomenon, with the VLA ${ }^{2}$ A-Array we observed an "incomplete"

\footnotetext{
* Images as FITS files are only available at the CDS via anonymous ftp to cdsarc.u-strasbg.fr $(130.79 .128 .5)$ or via http://cdsarc.u-strasbg.fr/viz-bin/qcat?]/A+A/555/A4

$1 \quad H_{0}=71 \mathrm{~km} \mathrm{~s}^{-1} \mathrm{Mpc}^{-1}, \Omega_{\mathrm{m}}=0.27, \Omega_{\mathrm{vac}}=0.73$.

2 The National Radio Astronomy Observatory is a facility of the National Science Foundation operated under cooperative agreement by Associated Universities, Inc.
}

sample of 29 sources selected from the list of Dallacasa (1990). The adopted selection criteria were: i) total flux density at $5 \mathrm{GHz}>1 \mathrm{Jy}$; ii) declination $>-20^{\circ}$; and iii) lack of observations at sub-arcsecond resolution (at the time of source selection).

In this paper, we report multi-frequency VLA polarisation observations of our CSS sample at 8.1, 8.5, 15 and $23 \mathrm{GHz}$.

In Sect. 2 we summarise the observations and data processing. Section 3 describes the new information obtained on the structural and polarisation properties. Discussion and conclusions are presented in Sects. 4 and 5, respectively.

\section{Observations and data reduction}

Polarimetric observations of our sample of 29 CSS sources were made at 8,15 and $23 \mathrm{GHz}$ on August 6th, 1991 using the VLA in A-array. The observations were scheduled in "bracket" mode, i.e. calibrator-source-calibrator, to obtain the best possible phase correction. Despite this, almost half of the data acquired at $23 \mathrm{GHz}$ could not be imaged due to poor weather conditions. The data were recorded in both circular polarisations, and calibrated in the standard way using AIPS procedures. The sources 2200+420 (BL Lac) and 0923+392 were observed regularly throughout the observing period to allow for parallactic angle corrections. Calibration of the Electric Vector Position Angle (EVPA) was performed by observing the source $1328+307$ (3C 286) assuming an EVPA of $33^{\circ}$ for it 

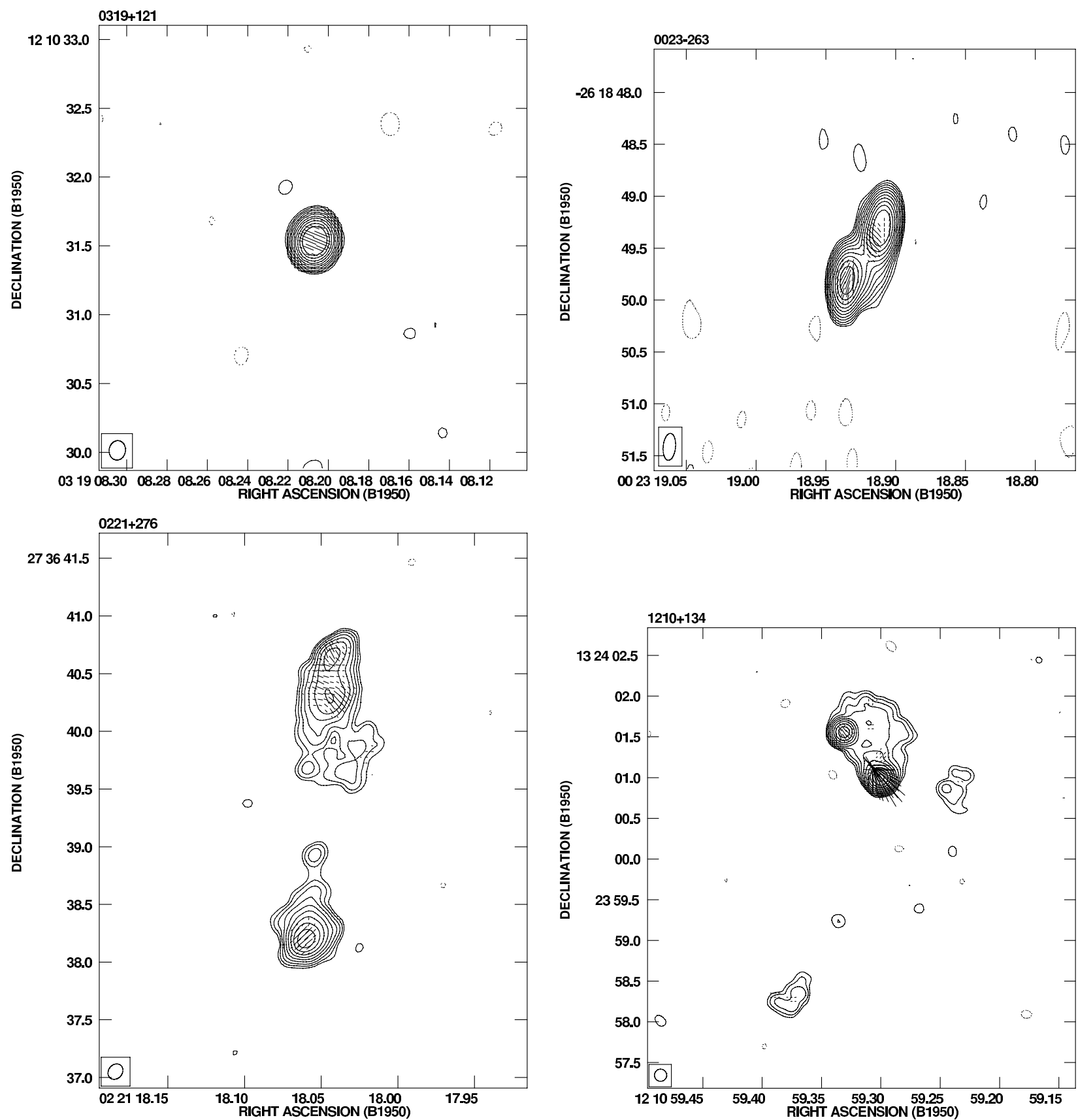

Fig. 1. Total-intensity images of a point-like source $(0319+121)$, a double source $(0023-263)$, a triple source $(0221+276)$, and a complex source $(1210+134)$ at $14885 \mathrm{MHZ}$. The peak flux density is $926.8 \mathrm{mJy} /$ beam, $635.8 \mathrm{mJy} / \mathrm{beam}, 96.8 \mathrm{mJy} / \mathrm{beam}, 161.3 \mathrm{mJy} / \mathrm{beam}$, respectively. Contours are $-1,1,2,4,8,16,32,64,128,256,512,1024 \mathrm{mJy} /$ beam. An electric vector length of 1 arcsec $=200 \mathrm{mJy} / \mathrm{beam}$ for $0319+121$, and $20 \mathrm{mJy} / \mathrm{beam}$ for others.

at all frequencies. An iterative procedure was carried out using the IMAGR and CALIB programmes to self-calibrate the parallel-hand (LL or RR) fringes. The complex gain corrections derived in this way were also applied to the cross-hand (RL or LR) fringes. Images in Stokes parameters $I, Q$, and $U$ were produced. Images of the polarised flux density $P=\left(Q^{2}+U^{2}\right)^{0.5}$ and EVPA, $\chi=0.5 \times \tan ^{-1}(U / Q)$, were then generated from the $Q$ and $U$ images. The data acquired with the $8 \mathrm{GHz}$ receiver were separately imaged for the two IFs, namely IF1 at $8085 \mathrm{MHz}$ and IF2 at $8485 \mathrm{MHz}$, each having a bandwidth of $50 \mathrm{MHz}$. When considered together, observations at these two frequencies are designated " $X$-band" in the text.

\section{Results}

\subsection{Images}

The full-resolution images are available online ${ }^{3}$.

In practice, half of the sources (15) are point-like at our resolution. However, two of these are slightly extended on one side. Seven others show double structure, and six more are triples. One further source is classified as complex. Examples of sources classified as above are presented in Fig. 1. This morphological description follows that of Spencer et al. (1991). The vectors representing the electric vector of linearly polarised intensity are overlaid on the total-intensity contours.

3 http://db.ira.inaf.it/aj206-fm/ 
F. Mantovani et al.: Radio polarimetry of compact steep spectrum sources at sub-arcsecond resolution
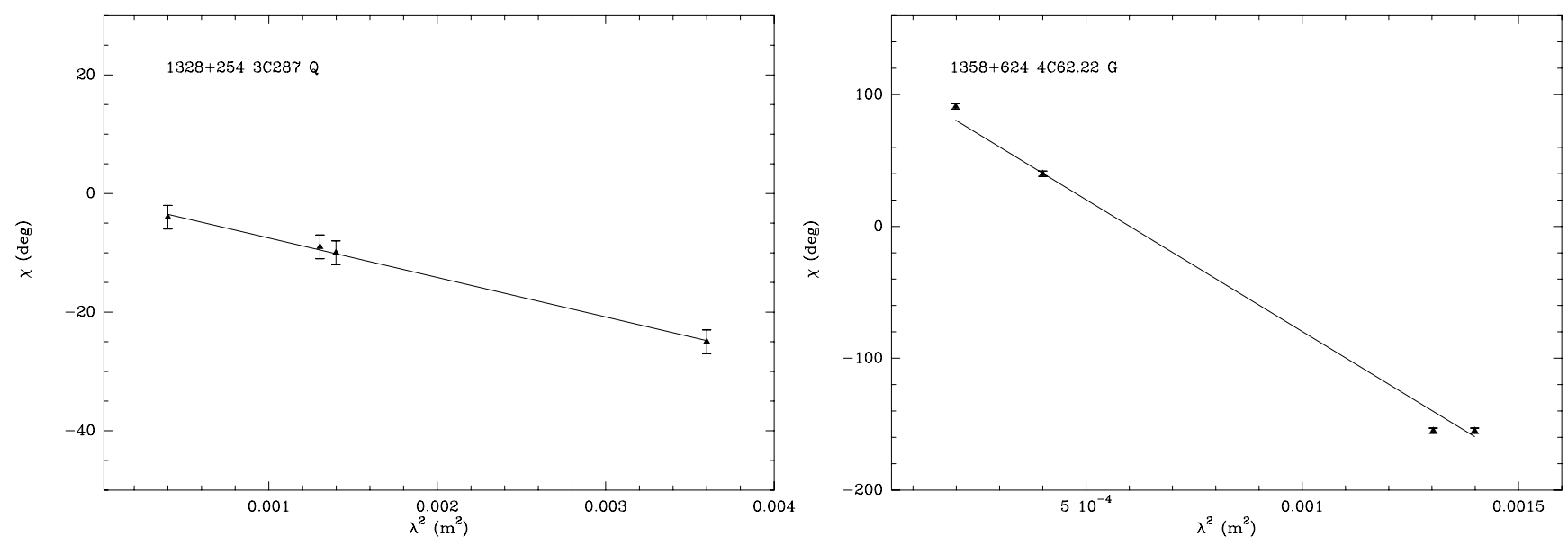

Fig. 2. Plots of the observed $\chi$ values of the quasar 1328+254 (3C 287) and of the galaxy 1358+624 (4C 62.22) as a function of $\lambda^{2}$ for the available frequencies.

Table 1. Percentage polarisations for the whole sample and for galaxies and quasars separately.

\begin{tabular}{lccc}
\hline \hline Frequency & \multicolumn{3}{c}{$\left\langle m_{\text {tot }}\right\rangle$} \\
MHz & all & $G$ & $Q$ \\
\hline 8085 & 1.1 & 0.5 & 2.8 \\
8485 & 1.1 & 0.3 & 2.8 \\
14885 & 3.0 & 1.1 & 4.2 \\
23285 & $\sim 3.5$ & $\sim 2.7$ & $\sim 4.0$ \\
\hline
\end{tabular}

Notes. Column 1: observing frequency; 2-4: the values of the median percentage polarisation $\left\langle m_{\mathrm{tot}}\right\rangle$ (the ratio of polarised flux density to the total flux density for the whole source) for the sample divided into galaxies $(G)$, and quasars $(Q)$.

\subsection{Polarised emission}

The great majority of the sample sources show evidence of polarised emission. However, generally, they are only weakly polarised. Three sources, namely $0531+194,0941-080$, and $1311+678$, show no polarised emission above the detection limits at any of the frequencies observed. The median percentage polarisations measured are summarised in Table 1. The polarisation percentage integrated over complete sources decreases from high to low frequencies, with quasars more polarised than galaxies. It could be noted that there are sources in which the polarisation percentage of individual components are higher than the polarisation percentage integrated over the complete source. This is due to averaging with a range of EVPAs.

The classification of source structures, together with percentage polarised emissions derived by integrating the total-intensity and the polarised-intensity emissions over the total extent of each source, and the depolarisation indices DP, defined as the ratio of the percentages of polarised emission at the lower to the higher frequencies, are reported in Table 2. In Tables 3-6, the polarisation parameters derived for the distinct components of each source at each observing frequency are reported. When more than one PA is listed in the Tables these refer to regions with different EVPAs. Further information may be obtained from the online images ${ }^{4}$.

Fractional polarisations, depolarisation indices, and rotation measure $(\mathrm{RM})$ values for the individual components of each

\footnotetext{
4 http://db.ira.inaf.it/aj206-fm/
}

source are reported in Table 7. The RM is defined as RM = $\Delta \chi / \Delta\left(\lambda^{2}\right)$ in $\operatorname{rad~m}^{-2}$ where $\chi$ is the PA at wavelength $\lambda$, allowing for $n \pi$ with $n$ an integer, in the individual PAs to get the best fit. When three frequencies are available, the ambiguity implied by the integer $n$ can be resolved. The RMs are estimated by fitting the points with a linear least-squares fit. The RM in the source rest frame is defined as $\mathrm{RM}_{\mathrm{rf}}=\mathrm{RM} \times(1+z)^{2}$.

We note that polarimetric parameters are derived from images at the full resolution achieved at the various frequencies. The $\chi$ values are those associated with the polarised emission peaks. The errors associated with the $\chi$ values used in the $\chi-\lambda^{2}$ plots are calculated considering the dispersion of $\chi$ values in boxes of $5 \times 5$ pixels around the positions of maximum polarised emission.

RM plots are also available online $e^{5}$. Examples of RM plots are presented in Fig. 2.

\subsection{Comments on individual sources}

Some of the CSS sources presented here have been included in other sub-arcsecond resolution observations. Parameters from those studies, and in particular the $\chi$ values, have been extracted when appropriate.

0023-263 (OB 238) - This source have been observed at the $\mathrm{S} / \mathrm{X}$ bands using VLBI by Tzioumis et al. (2002) with the SHEVE array. We confirm the double source structure of the source seen at both frequencies.

0114-211 (OC 224) - This source was observed with the VLA A-array at 5, 8.4, and $15 \mathrm{GHz}$ by Mantovani et al. (1994) who reported a triple structure. Polarised emission was detected only for the western component "c". The rest-frame RM was calculated in that paper assuming a conventional value of $z=0.5$. With the present $23.2 \mathrm{GHz}$ observations we detect total intensity and polarised emission only from component "c". Here we have used a redshift of $1.41 \pm 0.05$ (McCarthy et al. 1996) for estimating the rest-frame RM.

$0116+319$ (4C 31.04) - This source was observed by van Breugel et al. (1984) at $15 \mathrm{GHz}$ with the VLA. They detected no polarised emission above their detection limit of $1.5 \%$ of the flux density of $1226 \pm 16 \mathrm{mJy}$, which is a factor of $\sim 1.6$ higher than the flux density we find at the same frequency.

5 http://db.ira.inaf.it/aj206-fm/ 
Table 2. Integrated polarisation percentages for each source.

\begin{tabular}{|c|c|c|c|c|c|c|c|c|c|c|c|}
\hline Name & Other & ID & $z$ & rs & $m_{8.0}$ & $m_{8.4}$ & $m_{14.8}$ & $m_{23.2}$ & $D P_{8.0 / 8.4}$ & $D P_{8.4 / 14.8}$ & $D P_{14.8 / 23.2}$ \\
\hline B0023-263 & & G & 0.3216 & D & 0.4 & 0.27 & 0.67 & 1.5 & 1.48 & 0.40 & 0.45 \\
\hline B0114-211 & & G & 1.4100 & $\mathrm{Pe}$ & & & & 5.0 & & & \\
\hline B0116+319 & 4C 31.04 & G & 0.0602 & $\mathrm{P}$ & 0.27 & 0.18 & - & - & 1.50 & & \\
\hline B0127+233 & $3 \mathrm{C} 43$ & Q & 1.4590 & $\mathrm{~T}$ & 5.3 & 5.5 & 5.2 & 4.9 & 0.96 & 1.06 & 1.06 \\
\hline B0221+276 & $3 \mathrm{C} 67$ & $\mathrm{G}$ & 0.3102 & $\mathrm{~T}$ & 3.3 & 3.2 & 3.1 & & 1.03 & 1.02 & \\
\hline B0223+341 & 4C 34.07 & Q & 2.9100 & D & 0.43 & 0.34 & 0.58 & 0.85 & 1.26 & 0.59 & 0.68 \\
\hline В0319+121 & & Q & 2.6620 & $\mathrm{P}$ & 4.4 & 4.2 & 4.7 & & 1.05 & 0.89 & \\
\hline B0404+767 & 4C 76.03 & G & 0.5985 & $\mathrm{P}$ & 0.2 & 0.06 & 3.7 & & 3.3 & 0.016 & \\
\hline В0531+194 & & G & & D & - & - & - & & - & - & \\
\hline B $0538+498$ & 3C 147 & Q & 0.5450 & D & 0.93 & 0.98 & 2.9 & & 0.95 & 0.34 & \\
\hline В0941-080 & & G & 0.2280 & $\mathrm{P}$ & - & - & - & & - & - & \\
\hline B1005+077 & 3C 237 & G & 0.8770 & D & 0.63 & 0.73 & 3.6 & & 0.86 & 0.2 & \\
\hline B1151-348 & & Q & 0.2580 & $\mathrm{P}$ & 1.3 & 1.26 & 2.1 & & 1.03 & 0.6 & \\
\hline B1153+317 & 4C 31.38 & Q & 0.4170 & D & 2.1 & 2.1 & & & 1.0 & & \\
\hline B1210+134 & 4C 13.46 & Q & 1.1389 & $\mathrm{C}$ & 6.6 & 6.8 & 6.3 & & 0.97 & 1.07 & \\
\hline B1245-197 & & Q & 1.2750 & $\mathrm{Pe}$ & 0.07 & 0.15 & 0.57 & & 0.47 & 0.26 & \\
\hline B1311+678 & 4C 67.22 & G & & $\mathrm{P}$ & - & - & - & & - & - & \\
\hline B1323+321 & 4C 32.44 & $\mathrm{G}$ & 0.3680 & $\mathrm{P}$ & 0.78 & 0.93 & 1.1 & & 0.84 & 0.85 & \\
\hline B1328+254 & 3C 287 & Q & 1.0550 & $\mathrm{P}$ & 4.2 & 3.9 & 4.2 & & 1.08 & 0.93 & \\
\hline B1345+125 & 4C 12.50 & $\mathrm{G}$ & 0.1217 & $\mathrm{P}$ & 0.04 & 0.15 & 0.58 & & 0.27 & 0.26 & \\
\hline B1358+624 & 4C 62.22 & $\mathrm{G}$ & 0.4310 & $\mathrm{P}$ & 0.38 & 0.24 & 0.39 & - & 1.58 & 0.62 & \\
\hline B1416+067 & 3C 298 & Q & 1.4373 & $\mathrm{~T}$ & 2.6 & 2.5 & 3.0 & 3.2 & 1.04 & 0.83 & 0.94 \\
\hline B1458+718 & 3C 309.1 & Q & 0.9050 & $\mathrm{P}$ & 5.3 & 5.3 & 5.3 & 5.6 & 1.0 & 1.0 & 0.95 \\
\hline B1524-136 & & Q & 1.6870 & D & & & & 1.7 & & & \\
\hline B1634+628 & 3C 343 & G & 0.9880 & $\mathrm{P}$ & 1.2 & 1.2 & 1.3 & & 1.0 & 0.92 & \\
\hline B1638+124 & 4C 12.60 & G & 1.1520 & $\mathrm{P}$ & 0.21 & 0.21 & 0.63 & 1.7 & 1.0 & 0.33 & 0.37 \\
\hline B1641+173 & 3C 346 & G & 0.1620 & $\mathrm{~T}$ & 4.4 & 4.0 & 3.8 & 3.6 & 1.1 & 1.05 & 1.06 \\
\hline B1829+290 & 4C 29.56 & G & 0.8420 & $\mathrm{~T}$ & 0.6 & 0.50 & 0.8 & - & 1.20 & 0.62 & \\
\hline B2247+140 & 4C 14.82 & Q & 0.2346 & $\mathrm{P}$ & 2.9 & 3.0 & 4.5 & 4.5 & 0.97 & 0.67 & 1.0 \\
\hline
\end{tabular}

Notes. Column 1: source name; 2: other name; 3: optical identification; 4: redshift; 5: radio structure: P point-like, Pe point-like plus estension, D double, T triple, $\mathrm{C}$ complex; 6: polarisation percentage at $8085 \mathrm{MHz}$; : polarisation percentage at $8485 \mathrm{MHz}$; 8: polarisation percentage at $14885 \mathrm{MHz}$; 9: polarisation percentage at $23285 \mathrm{MHz}$; 10: depolarisation index, DP, between 8485 and 8085 MHz; 11: depolarisation index, DP, between 14885 and $8485 \mathrm{MHz}$; 12: depolarisation index, DP, between 23285 and $14885 \mathrm{MHz}$.

$0127+233(3 C 43)-$ The subject of many investigations, 3C 43 shows a peculiar structure characterized by a sharp bend of more than $90^{\circ}$ in the jet. Polarised emission has been reported by van Breugel et al. (1992), Akujor \& Garrington (1995), Sanghera et al. (1995), Lüdke et al. (1998) and Cotton et al. (2003b) at sub-arcsecond resolution. The EVPA in all these images made from data taken at different frequencies, is perpendicular to the jet axis, nicely following the bend in the jet. Small values of the RM are found along the jet region, while a high value of RM is measured for the northern component. VLBI observations were reported by Nan et al. (1991), Spencer et al. (1991), and Fanti et al. (2002). Polarimetric VLBI observations were performed by Mantovani et al. (2003) at $8.4 \mathrm{GHz}$. These authors detected polarised emission along the jet with polarisation percentages up to $12 \%$. However, no polarisation was detected from the region of the core. A similar result was obtained at $1.6 \mathrm{GHz}$ by Cotton et al. (2003a). Both articles report a large $\mathrm{RM}$ near the bend in the jet.

$0221+276$ (3C 67) - This source shows a triple structure from our $15 \mathrm{GHz}$ observations, as did MERLIN observations at $5 \mathrm{GHz}$ by Sanghera et al. (1995). Because of its almost-flat spectrum, we confirm that the central weak component close to the southern lobe is the core of the source, as suggested by Sanghera et al. (1995) and Lüdke et al. (1998). Of the two lobes, that to the north is strongly polarised at the frequencies we observed. The area with the highest polarised emission shows RMs of up to
$1620 \mathrm{rad} \mathrm{m}^{-2}$ in the source rest frame. The southern lobe is $2 \%$ polarised at $15 \mathrm{GHz}$, and depolarises quickly towards lower frequencies. Polarised emission from this component is only marginally detected by our $X$-band observations. The RM value ( $\sim 3000 \mathrm{rad} \mathrm{m}^{-2}$ in the source rest-frame) calculated for this component is rather uncertain.

$0223+341$ (4C 34.07) - The images at 15 and $23.2 \mathrm{GHz}$ reveal a triple structure. The central component is unpolarised and has an almost flat spectrum between the two frequencies. In contrast, both lobes are polarised. However, the less-polarised northern component shows depolarisation between 23.2 and $15 \mathrm{GHz}$, but a constant polarisation percentage between 15 and $8.0 \mathrm{GHz}$. The source rest-frame RM value found for this component of more than $-36000 \mathrm{rad} \mathrm{m}^{-2}$ is calculated after adding $180^{\circ}$ to $\chi$ at $23.3 \mathrm{GHz}$. Subtracting $180^{\circ}$ from $\chi$ at 8.0 and $8.4 \mathrm{GHz}$ will generate an even higher RM. Both solutions give acceptable fits to the $\lambda^{2}$ law. If we do not adjust any of the above three $\chi$ values, a rare case is implied in which the $\chi$ values do not obey the $\lambda^{2}$ law.

The southern component is strongly polarised at $15 \mathrm{GHz}$, while the polarisation percentage is a factor of 5 lower at $X$-band. An $R M$ value of $R M=5871 \mathrm{rad} \mathrm{m}^{-2}$ in the source rest-frame can be unambiguosly derived.

$0319+121$ - This source is point-like at $15 \mathrm{GHz}$, but shows an extension to the north at $X$-band. VLBI S/X band images from the US Naval Observatory Radio Reference Frame Image 
F. Mantovani et al.: Radio polarimetry of compact steep spectrum sources at sub-arcsecond resolution

Table 3. Polarisation parameters for components of each source at $8085 \mathrm{MHz}$.

\begin{tabular}{|c|c|c|c|c|c|c|c|c|c|c|c|}
\hline Name & Other name & ID & Comp & $\begin{array}{r}S \\
\mathrm{mJy}\end{array}$ & $\begin{array}{l}3 \sigma \\
\mathrm{mJy} / \mathrm{b}\end{array}$ & $\begin{array}{l}S p \\
\text { mJy }\end{array}$ & $\begin{array}{l}3 \sigma p \\
\mathrm{mJy} / \mathrm{b}\end{array}$ & $\begin{array}{c}\chi \\
\text { deg }\end{array}$ & $\begin{array}{l}\text { Maj } \\
\text { arcsec }\end{array}$ & $\begin{array}{l}\text { Min } \\
\operatorname{arcsec}\end{array}$ & $\begin{array}{r}\text { PA } \\
\text { deg }\end{array}$ \\
\hline \multirow[t]{2}{*}{ B0023-263 } & & $\mathrm{G}$ & $\mathrm{a}$ & 1329.2 & 0.29 & 5.7 & 0.15 & 53 & 0.16 & 0.05 & 158 \\
\hline & & & b & 848.2 & & 3.3 & & 49 & 0.11 & 0.06 & 129 \\
\hline B0116+319 & 4C 31.04 & $\mathrm{G}$ & & 1097.8 & 0.22 & 3.0 & 0.11 & -4 & 0.10 & 0.02 & 116 \\
\hline \multirow[t]{3}{*}{ B $0127+233$} & $3 \mathrm{C} 43$ & Q & $a_{12}$ & 637.2 & 0.21 & $12.8 / 16.0$ & 0.12 & $80 / 13$ & 0.21 & 0.11 & 148 \\
\hline & & & $\mathrm{b}$ & 114.6 & & 10.7 & & -33 & 1.1 & 0.2 & 59 \\
\hline & & & $\mathrm{c}$ & 24.6 & & 1.4 & & 44 & 0.33 & 0.26 & 139 \\
\hline \multirow[t]{2}{*}{ B0221+276 } & $3 \mathrm{C} 67$ & G & $\mathrm{a}$ & 228.8 & 0.08 & 19.6 & 0.09 & $50-80$ & 0.58 & 0.19 & 173 \\
\hline & & & $\mathrm{b}$ & 357.4 & & & & & 0.17 & 0.13 & 176 \\
\hline \multirow[t]{2}{*}{ B0223+341 } & 4C 34.07 & Q & $\mathrm{a}$ & 1254.0 & 0.18 & 4.6 & 0.12 & -6 & 0.03 & 0.02 & 43 \\
\hline & & & $\mathrm{b}$ & 66.2 & & 1.1 & & -54 & 0.22 & 0.07 & 81 \\
\hline В0319+121 & & & & 1228.1 & 0.15 & 54.0 & 0.10 & 67 & 0.03 & 0.003 & 158 \\
\hline В0404+767 & 4C 76.03 & $\mathrm{E}$ & & 2194.9 & 0.27 & 4.4 & 0.14 & -41 & 0.076 & 0.013 & 43 \\
\hline \multirow[t]{2}{*}{ B0531+194 } & & G & a & 703.7 & 0.15 & & 0.09 & & 0.19 & 0.09 & 139 \\
\hline & & & $\mathrm{b}$ & 751.3 & & & & & 0.23 & 0.09 & 123 \\
\hline \multirow[t]{2}{*}{ B0538+498 } & 3C 147 & Q & $\mathrm{a}$ & 4474.6 & 0.36 & 32.2 & 0.10 & -23 & 0.33 & 0.26 & 57 \\
\hline & & & $\mathrm{b}$ & 393.6 & & 12.5 & & -70 & 0.32 & 0.27 & 24 \\
\hline В0941-080 & & G & & 706.0 & 0.12 & & 0.09 & & 0.046 & 0.01 & 144 \\
\hline \multirow[t]{2}{*}{ B1005+077 } & $3 C 237$ & G & a & 684.7 & 0.12 & 6.5 & 0.09 & 28 & 0.16 & 0.06 & 78 \\
\hline & & & b & 462.6 & & 0.7 & & -52 & 0.15 & 0.06 & 87 \\
\hline B1151-348 & & Q & & 1749.8 & 0.21 & 22.7 & 0.31 & 20 & 0.11 & 0.05 & 60 \\
\hline \multirow[t]{2}{*}{ B1153+317 } & 4C 31.38 & $\mathrm{Q}$ & $\mathrm{a}$ & 400.0 & 0.08 & 3.4 & 0.09 & -62 & 0.11 & 0.06 & 8 \\
\hline & & & $\mathrm{b}$ & 214.9 & & 9.7 & & 22 & 0.11 & 0.04 & 48 \\
\hline \multirow[t]{6}{*}{ B1210+134 } & 4C 13.46 & Q & $\mathrm{a}$ & 307.5 & 0.08 & 22.0 & 0.09 & 45 & 0.15 & 0.09 & 15 \\
\hline & & & b & 200.0 & & 8.4 & & 33 & 0.21 & 0.13 & 135 \\
\hline & & & $\mathrm{c}$ & 1.8 & & & & & 0.68 & 0.19 & 125 \\
\hline & & & d & 8.8 & & 1.8 & & 55 & 0.28 & 0.22 & 165 \\
\hline & & & $\mathrm{e}$ & 6.5 & & 0.2 & & -42 & 0.65 & 0.35 & 1 \\
\hline & & & $\mathrm{f}$ & 15.3 & & 3.3 & & $-84 /-57$ & 0.48 & 0.21 & 134 \\
\hline B1245-197 & & Q & & 1553.2 & 0.21 & 0.07 & 0.14 & 58 & 0.09 & 0.02 & 87 \\
\hline B1311+678 & 4C 67.22 & $\mathrm{E}$ & & 608.1 & 0.06 & & 0.09 & & 0.06 & 0.02 & 112 \\
\hline B1323+321 & $4 C 32.44$ & $\mathrm{G}$ & & 1620.9 & 0.20 & 12.6 & 0.09 & 7 & 0.06 & 0.01 & 134 \\
\hline B1328+254 & $3 \mathrm{C} 287$ & $\mathrm{Q}$ & & 2239.6 & 0.30 & 92.9 & 0.10 & -10 & 0.06 & 0.04 & 25 \\
\hline B1345+125 & $4 C 12.50$ & $\mathrm{G}$ & & 2291.7 & 0.40 & 1.0 & 0.10 & 42 & 0.06 & 0.015 & 170 \\
\hline B1358+624 & $4 \mathrm{C} 62.22$ & $\mathrm{G}$ & & 1182.9 & 0.05 & 4.5 & 0.18 & 25 & 0.03 & 0.01 & 124 \\
\hline \multirow[t]{3}{*}{ B1416+067 } & $3 \mathrm{C} 298$ & Q & $a+b$ & 304.1 & 0.08 & $12.1 / 11.0$ & 0.12 & $-55 /-7$ & 0.25 & 0.12 & 85 \\
\hline & & & $\mathrm{c}$ & 215.2 & & & & & 0.03 & 0.0 & 46 \\
\hline & & & $\mathrm{d}$ & 388.7 & & & & & 0.69 & 0.13 & 102 \\
\hline \multirow[t]{5}{*}{ B1458+718 } & 3C 309.1 & Q & $\mathrm{a} / \mathrm{a}_{1}$ & 359.9 & 0.33 & $15.4 / 11.8$ & 0.15 & $-70 /-16$ & 0.41 & 0.18 & 116 \\
\hline & & & $\mathrm{b}$ & 1598.0 & & 80.2 & & 49 & 0.087 & 0.044 & 76 \\
\hline & & & $\mathrm{c}$ & 26.4 & & 0.6 & & 78 & 0.45 & 0.27 & 67 \\
\hline & & & d & 83.3 & & 1.5 & & -48 & 0.36 & 0.29 & 99 \\
\hline & & & $\mathrm{e}$ & 32.1 & & 0.8 & & -44 & 0.63 & 0.39 & 162 \\
\hline \multirow[t]{2}{*}{ B1634+628 } & $3 \mathrm{C} 343$ & $\mathrm{G}$ & $\mathrm{a}$ & 825.3 & 0.14 & 9.9 & 0.10 & 80 & 0.09 & 0.06 & 94 \\
\hline & & & $\mathrm{b}$ & 5.4 & & & & & 0.79 & 0.28 & 59 \\
\hline B1638+124 & 4C 12.60 & $\mathrm{E}$ & & 788.1 & 0.13 & 1.72 & 0.09 & -77 & 0.14 & 0.012 & 130 \\
\hline \multirow[t]{3}{*}{ B1641+173 } & $3 \mathrm{C} 346$ & $\mathrm{G}$ & $a_{123}$ & 331.4 & 0.10 & $0.7 / 17.3 / 7.6$ & 0.09 & $0 /-36 / 1$ & - & - & - \\
\hline & & & $\mathrm{b}$ & 205.3 & & & & & 0.015 & 0.0 & 1 \\
\hline & & & $\mathrm{c}$ & 40.7 & & & & & 3.11 & 0.9 & 54 \\
\hline \multirow[t]{4}{*}{ B1829+290 } & 4C 29.56 & G & $\mathrm{a}$ & 11.4 & 0.10 & & 0.10 & & 0.62 & 0.22 & 88 \\
\hline & & & $c+b$ & 685.1 & & 4.2 & & -28 & 0.077 & 0.014 & 86 \\
\hline & & & $\mathrm{d}$ & 2.2 & & & & & 0.40 & 0.04 & 62 \\
\hline & & & $\mathrm{e}$ & 10.3 & & 0.3 & & -45 & 0.61 & 0.27 & 56 \\
\hline B $2247+140$ & 4C 14.82 & Q & $a+b$ & 833.1 & 0.21 & $17.1 / 7.1$ & 0.12 & $-78 / 15$ & 0.21 & 0.07 & 31 \\
\hline
\end{tabular}

Notes. Column 1: source name; 2: other name; 3: optical identification; 4: component; 5: integrated component flux density; 6: three $\sigma$ noise in total-intensity image; 7: polarised flux density; 8: three $\sigma$ noise in the polarised-intensity density; 9: EVPA; 10-12: deconvolved sizes of the components; major axis, minor axis, and position angle of the major axis, respectively.

Database $^{6}$ show a core-jet structure, with the jet in the same direction we find. The source presents little depolarisation from higher to lower frequencies and an RM of zero.

0404+767 (4C 76.03) - Again a point-like source at subarcsecond resolution. In this case there is a strong depolarisation between 15 and $8.0 \mathrm{GHz}$. However, the percentage polarisation at $8.4 \mathrm{GHz}$ is much lower that at $8.0 \mathrm{GHz}$, which is possibly due to uncertainties caused by the low levels of polarisation. Nevertheless, the $\chi$ values fit the $\lambda^{2}$ law nicely, with $\mathrm{RM}=5350 \mathrm{rad} \mathrm{m}^{-2}$ in the source rest-frame. The VLBI image

\footnotetext{
${ }^{6}$ http://rorf.usno.navy.mil/RRFID/
} 
A\&A 555, A4 (2013)

Table 4. Polarisation parameters for components of each source at $8485 \mathrm{MHz}$.

\begin{tabular}{|c|c|c|c|c|c|c|c|c|c|c|c|}
\hline Name & Other name & ID & Comp & $\begin{array}{r}S \\
\mathrm{mJy}\end{array}$ & $\begin{array}{l}3 \sigma \\
\mathrm{mJy} / \mathrm{b}\end{array}$ & $\begin{array}{l}S p \\
\text { mJy }\end{array}$ & $\begin{array}{l}3 \sigma p \\
\mathrm{mJy} / \mathrm{b}\end{array}$ & $\begin{array}{c}x \\
\text { deg }\end{array}$ & $\begin{array}{l}\text { Maj } \\
\text { arcsec }\end{array}$ & $\begin{array}{l}\text { Min } \\
\operatorname{arcsec}\end{array}$ & $\begin{array}{r}\text { PA } \\
\text { deg }\end{array}$ \\
\hline \multirow[t]{2}{*}{ B0023-263 } & & G & $\mathrm{a}$ & 1261.8 & 0.30 & 3.8 & 0.15 & 63 & 0.16 & 0.05 & 157 \\
\hline & & & b & 893.7 & & 2.0 & & 59 & 0.12 & 0.06 & 133 \\
\hline B0116+319 & 4C 31.04 & $\mathrm{G}$ & & 1070.8 & 0.30 & 1.97 & 0.10 & -4 & 0.09 & 0.019 & 116 \\
\hline \multirow{3}{*}{ B $0127+233$} & $3 \mathrm{C} 43$ & $\mathrm{Q}$ & a12 & 619.2 & 0.11 & $6.4 / 20.7$ & 0.11 & $80 / 13$ & 0.21 & 0.11 & 147 \\
\hline & & & $\mathrm{b}$ & 98.4 & & 11.9 & & -34 & 1.0 & 0.18 & 58 \\
\hline & & & $\mathrm{c}$ & 25.5 & & 1.9 & & 44 & 0.43 & 0.29 & 136 \\
\hline \multirow[t]{2}{*}{ B $0221+276$} & $3 \mathrm{C} 67$ & G & $\mathrm{a}$ & 219.0 & 0.07 & 17.8 & 0.08 & $50-80$ & 0.58 & 0.18 & 173 \\
\hline & & & $\mathrm{b}$ & 339.6 & & & & & 0.16 & 0.13 & 175 \\
\hline \multirow[t]{2}{*}{ B0223+341 } & 4C 34.07 & Q & $\mathrm{a}$ & 1225.6 & 0.11 & 3.6 & 0.09 & -4 & 0.03 & 0.014 & 54 \\
\hline & & & b & 55.2 & & 0.8 & & -55 & 0.019 & 0.085 & 77 \\
\hline B0319+121 & & & & 1211.8 & 0.15 & 50.9 & 0.12 & 67 & 0.03 & 0.002 & 160 \\
\hline В0404+767 & 4C 76.03 & $\mathrm{E}$ & & 2124.2 & 0.16 & 1.26 & 0.13 & -60 & 0.076 & 0.007 & 43 \\
\hline \multirow[t]{2}{*}{ B $0531+194$} & & $\mathrm{G}$ & $\mathrm{a}$ & 624.3 & 0.15 & 0.8 & 0.08 & -45 & 0.23 & 0.09 & 136 \\
\hline & & & $\mathrm{b}$ & 719.3 & & 0.4 & & -45 & 0.21 & 0.09 & 122 \\
\hline \multirow{2}{*}{ B0538+498 } & 3C 147 & Q & $\mathrm{a}$ & 4258.0 & 0.39 & 31.6 & 0.15 & -18 & 0.22 & 0.06 & 55 \\
\hline & & & $\mathrm{b}$ & 370.3 & & 13.6 & & -75 & 0.40 & 0.10 & 18 \\
\hline В0941-080 & & $\mathrm{G}$ & & 673.5 & 0.12 & & 0.11 & & 0.06 & 0.009 & 142 \\
\hline \multirow[t]{2}{*}{ B1005+077 } & 3C 237 & $\mathrm{G}$ & $\mathrm{a}$ & 659.9 & 0.12 & 6.9 & 0.09 & 34 & 0.16 & 0.06 & 78 \\
\hline & & & b & 439.0 & & 1.1 & & -52 & 0.16 & 0.07 & 88 \\
\hline B1151-348 & & Q & & 1678.7 & 0.81 & 21.0 & 0.39 & 26 & 0.11 & 0.04 & 60 \\
\hline \multirow[t]{2}{*}{ B1153+317 } & 4C 31.38 & Q & $\mathrm{a}$ & 383.1 & 0.06 & 2.6 & 0.08 & -66 & 0.10 & 0.06 & 5 \\
\hline & & & $\mathrm{b}$ & 203.3 & & 9.6 & & 22 & 0.10 & 0.04 & 48 \\
\hline \multirow[t]{6}{*}{ B1210+134 } & 4C 13.46 & Q & $\mathrm{a}$ & 297.4 & 0.07 & 20.7 & 0.11 & 49 & 0.15 & 0.10 & 11 \\
\hline & & & b & 177.4 & & 8.1 & & 34 & 0.20 & 0.12 & 112 \\
\hline & & & $\mathrm{c}$ & 10.2 & & & & & - & - & - \\
\hline & & & d & 10.1 & & 2.1 & & 62 & 0.33 & 0.27 & 2 \\
\hline & & & $\mathrm{e}$ & 3.9 & & 0.3 & & -46 & 0.45 & 0.35 & 176 \\
\hline & & & $\mathrm{f}$ & 14.7 & & 3.7 & & $-84 /-57$ & 0.57 & 0.24 & 143 \\
\hline B1245-197 & & Q & & 1486.7 & 0.17 & 2.2 & 0.13 & -76 & 0.088 & 0.016 & 87 \\
\hline B1311+678 & 4C 67.22 & $\mathrm{E}$ & & 583.1 & 0.10 & & 0.08 & & 0.06 & 0.02 & 113 \\
\hline B1323+321 & $4 C 32.44$ & $\mathrm{G}$ & & 1566.8 & 0.21 & 14.6 & 0.11 & 15 & 0.06 & 0.013 & 133 \\
\hline B1328+254 & $3 \mathrm{C} 287$ & $\mathrm{Q}$ & & 2167.2 & 0.33 & 85.4 & 0.19 & -9 & 0.064 & 0.039 & 23 \\
\hline B $1345+125$ & $4 C 12.50$ & $\mathrm{G}$ & & 2215.9 & 0.51 & 3.3 & 0.11 & -81 & 0.063 & 0.013 & 170 \\
\hline B1358+624 & $4 \mathrm{C} 62.22$ & $\mathrm{G}$ & & 1136.5 & 0.14 & 2.7 & 0.12 & 25 & 0.036 & 0.007 & 119 \\
\hline \multirow[t]{3}{*}{ B $1416+067$} & $3 C 298$ & $\mathrm{Q}$ & $a+b$ & 286.0 & 0.09 & $11.3 / 10.4$ & 0.07 & $-57 /-6$ & 0.25 & 0.12 & 85 \\
\hline & & & $\mathrm{c}$ & 210.4 & & & & & 0.04 & 0.0 & 47 \\
\hline & & & $\mathrm{d}$ & 357.2 & & & & & 0.68 & 0.14 & 103 \\
\hline \multirow[t]{5}{*}{ B1458+718 } & 3 C 309.1 & $\mathrm{Q}$ & $\mathrm{a} / \mathrm{a} 1$ & 348.0 & 0.28 & $14.1 / 11.4$ & 0.15 & $-64 /-18$ & 0.42 & 0.18 & 116 \\
\hline & & & $\mathrm{b}$ & 1543.1 & & 78.0 & & 49 & 0.080 & 0.044 & 78 \\
\hline & & & $\mathrm{c}$ & 28.7 & & & & 78 & 0.53 & 0.33 & 172 \\
\hline & & & d & 75.9 & & 1.6 & & -48 & 0.36 & 0.24 & 96 \\
\hline & & & $\mathrm{e}$ & 41.4 & & & & -44 & 1.00 & 0.54 & 28 \\
\hline \multirow[t]{2}{*}{ B1634+628 } & $3 \mathrm{C} 343$ & $\mathrm{G}$ & $\mathrm{a}$ & 778.1 & 0.16 & 9.1 & 0.09 & 77 & 0.083 & 0.062 & 100 \\
\hline & & & $\mathrm{b}$ & 3.3 & & & & & 0.62 & 0.33 & 123 \\
\hline B1638+124 & 4C 12.60 & $\mathrm{E}$ & & 757.0 & 0.10 & 1.60 & 0.08 & -77 & 0.14 & 0.02 & 130 \\
\hline \multirow{3}{*}{ B1641+173 } & $3 C 346$ & $\mathrm{G}$ & a123 & 311.6 & 0.06 & $0.0 / 15.5 / 7.6$ & 0.07 & $-1-36 / 0$ & - & - & - \\
\hline & & & $\mathrm{b}$ & 206.7 & & & & & 0.007 & 0.00 & 37 \\
\hline & & & $\mathrm{c}$ & 43.6 & & & & & 1.41 & 0.82 & 95 \\
\hline \multirow[t]{4}{*}{ B1829+290 } & 4C 29.56 & G & $\mathrm{a}$ & 9.2 & 0.07 & & 0.09 & & 0.45 & 0.22 & 87 \\
\hline & & & $c+b$ & 652.5 & & 3.2 & & -30 & 0.07 & 0.010 & 87 \\
\hline & & & $\mathrm{d}$ & 1.6 & & & & & 0.28 & 0.09 & 49 \\
\hline & & & $\mathrm{e}$ & 11.0 & & 0.2 & & -45 & 0.58 & 0.52 & 28 \\
\hline B $2247+140$ & 4C 14.82 & Q & $a+b$ & 794.9 & 0.36 & $17.2 / 6.8$ & 0.08 & $-79 / 15$ & 0.21 & 0.05 & 31 \\
\hline
\end{tabular}

Notes. Column 1: source name; 2: other name; 3: optical identification; 4: component; 5: integrated flux density of the components; 6: three $\sigma$ error on the total-intensity image; 7: polarised flux density; 8: three $\sigma$ error on the polarised-intensity image; 9: EVPA; 10-12: deconvolved sizes of the components; major axis, minor axis, and position angle of the major axis, respectively.

at $8.0 \mathrm{GHz}$ by $\mathrm{Xu}$ et al. (1995) shows a triple structure for 0404+767 at milli-arcsecond resolution.

$0531+194-$ A compact double source that is unpolarised at the detection levels of all our observations.

$0538+498$ (3C 147) - Our results for this source are fully described in Junor et al. (1999).
0941-080 - A point-like source with polarised emission below the detection limits at all of the available frequencies.

1005-077 (3C 237) - The triple structure seen by Lüdke et al. (1998) observing 3C 237 with MERLIN at $5 \mathrm{GHz}$ is confirmed by our $15 \mathrm{GHz}$ observations. The central component has a flat spectral index between 5 and $15 \mathrm{GHz}$, has no detectable 
F. Mantovani et al.: Radio polarimetry of compact steep spectrum sources at sub-arcsecond resolution

Table 5. Polarisation parameters for components of each source at $14885 \mathrm{MHz}$.

\begin{tabular}{|c|c|c|c|c|c|c|c|c|c|c|c|}
\hline Name & Other name & ID & Comp & $\begin{array}{c}S \\
\text { mJy }\end{array}$ & $\begin{array}{l}3 \sigma \\
\mathrm{mJy} / \mathrm{b}\end{array}$ & $\begin{array}{l}S p \\
\text { mJy }\end{array}$ & $\begin{array}{l}3 \sigma p \\
\mathrm{mJy} / \mathrm{b}\end{array}$ & $\begin{array}{c}\chi \\
\text { deg }\end{array}$ & $\begin{array}{l}\text { Maj } \\
\text { arcsec }\end{array}$ & $\begin{array}{l}\text { Min } \\
\operatorname{arcsec}\end{array}$ & $\begin{array}{l}\text { PA } \\
\text { deg }\end{array}$ \\
\hline \multirow[t]{2}{*}{ B0023-263 } & & $\mathrm{G}$ & a12 & 747.70 & 0.54 & $2.9 / 2.1$ & 0.45 & $45 /-14$ & 0.11 & 0.03 & 150 \\
\hline & & & $\mathrm{b}$ & 509.30 & & 3.4 & & -23 & 0.089 & 0.038 & 129 \\
\hline B0116+319 & 4C 31.04 & $\mathrm{G}$ & & 746.30 & 0.33 & & 0.38 & & 0.098 & 0.025 & 114 \\
\hline \multirow[t]{4}{*}{ B $0127+233$} & $3 \mathrm{C} 43$ & Q & a1 & 241.80 & 0.30 & 11.4 & 0.34 & 14 & 0.13 & 0.05 & 86 \\
\hline & & & a2 & 162.00 & & 11.7 & & 81 & 0.12 & 0.0 & 2 \\
\hline & & & $\mathrm{b}$ & 27.90 & & & & & 0.53 & 0.16 & 55 \\
\hline & & & $\mathrm{c}$ & 8.48 & & & & & 0.28 & 0.07 & 37 \\
\hline \multirow[t]{4}{*}{ B0221+276 } & $3 \mathrm{C} 67$ & G & a12 & 100.7 & 0.21 & 5.4 & 0.23 & 25 & 0.61 & 0.164 & 172 \\
\hline & & & a3 & 8.6 & & & & & - & - & - \\
\hline & & & $\mathrm{b}$ & 178.0 & & 3.6 & & -49 & 0.14 & 0.12 & 168 \\
\hline & & & $\mathrm{c}$ & 1.24 & & & & & 0.06 & 0.026 & 168 \\
\hline \multirow[t]{3}{*}{ B $0223+341$} & 4C 34.07 & Q & $\mathrm{a}$ & 845.40 & 0.21 & 3.2 & 0.30 & -70 & 0.017 & 0.006 & 108 \\
\hline & & & $\mathrm{b}$ & 25.7 & & 1.9 & & -75 & 0.158 & 0.055 & 88 \\
\hline & & & b0 & 3.38 & & & & & 0.25 & 0.04 & 59 \\
\hline В0319+121 & & & & 934.80 & 0.27 & 43.6 & 0.28 & 67 & 0.018 & 0.005 & 164 \\
\hline B0404+767 & 4C 76.03 & E & & 1496.00 & 0.27 & 54.7 & 0.32 & 25 & 0.06 & 0.010 & 46 \\
\hline \multirow[t]{2}{*}{ B0531+194 } & & $\mathrm{G}$ & a & 294.20 & 0.33 & & 0.30 & & 0.124 & 0.084 & 138 \\
\hline & & & $\mathrm{b}$ & 365.90 & & & & & 0.15 & 0.077 & 123 \\
\hline \multirow[t]{3}{*}{ B $0538+498$} & $3 \mathrm{C} 147$ & Q & a1 & 1409.70 & 0.42 & 58.1 & 0.42 & 50 & 0.06 & 0.0 & 105 \\
\hline & & & a2 & 1029.40 & & 2.9 & & 45 & 0.12 & 0.06 & 63 \\
\hline & & & $\mathrm{b}$ & 178.10 & & 15.1 & & 90 & 0.21 & 0.08 & 16 \\
\hline В0941-080 & & G & & 390.40 & 0.24 & & & & 0.065 & 0.008 & 143 \\
\hline \multirow{3}{*}{ B1005+077 } & 3 C 237 & G & $\mathrm{a}$ & 336.20 & 0.18 & 15.9 & 0.30 & $2 / 42$ & 0.16 & 0.04 & 78 \\
\hline & & & $\mathrm{b}$ & 215.00 & & 4.0 & & 0 & 0.12 & 0.05 & 85 \\
\hline & & & b1 & 3.86 & & & & & 0.14 & 0.04 & 105 \\
\hline B1151-348 & & Q & & 966.40 & 0.68 & 20.0 & 0.64 & 9 & 0.10 & 0.04 & 68 \\
\hline \multirow[t]{5}{*}{ B1210+134 } & 4C 13.46 & Q & $\mathrm{a}$ & 205.8 & 0.18 & 20.2 & 0.30 & 40 & 0.08 & 0.05 & 42 \\
\hline & & & $\mathrm{b}$ & 116.3 & & 2.3 & & 35 & 0.04 & 0.03 & 21 \\
\hline & & & c & $26.4 ?$ & & & & & - & - & - \\
\hline & & & d & 3.20 & & & & & 0.49 & 0.23 & 137 \\
\hline & & & $\mathrm{f}$ & 5.30 & & & & & 0.40 & 0.15 & 141 \\
\hline \multirow[t]{2}{*}{ B1245-197 } & & Q & $\mathrm{a}$ & 804.9 & 0.24 & 5.0 & 0.32 & 52 & 0.0 & 0.0 & \\
\hline & & & $\mathrm{b}$ & 71.8 & & & & & 0.11 & 0.02 & 102 \\
\hline B1311+678 & $4 \mathrm{C} 67.22$ & $\mathrm{E}$ & & 328.30 & 0.15 & & 0.32 & & 0.05 & 0.02 & 113 \\
\hline B1323+321 & 4C 32.44 & $\mathrm{G}$ & & 1038.00 & 0.27 & 11.4 & 0.19 & 11 & 0.06 & 0.010 & 132 \\
\hline B1328+254 & $3 C 287$ & Q & & 1389.0 & 0.42 & 58.8 & 0.25 & -4 & 0.05 & 0.03 & 28 \\
\hline B $1345+125$ & $4 \mathrm{C} 12.50$ & $\mathrm{G}$ & & 1535.0 & 0.18 & 8.9 & 0.25 & 20 & 0.06 & 0.01 & 171 \\
\hline B $1358+624$ & $4 C 62.22$ & $\mathrm{G}$ & & 721.04 & 0.45 & 2.8 & 0.32 & 45 & 0.029 & 0.011 & 96 \\
\hline \multirow{5}{*}{ B1416+067 } & $3 C 298$ & $\mathrm{Q}$ & $\mathrm{a}$ & 85.40 & 0.25 & 6.1 & 0.32 & -63 & 0.12 & 0.07 & 33 \\
\hline & & & $\mathrm{b}$ & 46.3 & & 6.1 & & -2 & 0.19 & 0.07 & 71 \\
\hline & & & $\mathrm{c} 1$ & 70.50 & & 0.7 & & -1 & 0.11 & 0.03 & 96 \\
\hline & & & $\mathrm{c} 2$ & 167.20 & & & & & 0.014 & 0.0 & 54 \\
\hline & & & $\mathrm{d}$ & 60.10 & & & & & 0.08 & 0.07 & 41 \\
\hline \multirow[t]{5}{*}{ B1458+718 } & 3C 309.1 & Q & $\mathrm{a}$ & 76.60 & 0.36 & 7.7 & 0.43 & $-87 /-46$ & 0.16 & 0.0 & 13 \\
\hline & & & $\mathrm{a} 0$ & 104.7 & & & & & 0.39 & 0.21 & 110 \\
\hline & & & a1 & 38.3 & & 3.3 & & -23 & 0.022 & 0.096 & 95 \\
\hline & & & $\mathrm{b}$ & 1051.90 & & 58.2 & & 45 & 0.048 & 0.025 & 146 \\
\hline & & & $\mathrm{d}$ & 28.00 & & & & & 0.28 & 0.15 & 89 \\
\hline B1634+628 & 3C 343 & $\mathrm{G}$ & $\mathrm{a}$ & 376.00 & 0.18 & 5.1 & 0.32 & 42 & 0.07 & 0.05 & 87 \\
\hline \multirow[t]{2}{*}{ B1638+124 } & 4C 12.60 & $\mathrm{E}$ & $\mathrm{a}$ & 432.10 & 0.23 & 0.7 & 0.30 & -1 & 0.02 & 0.01 & 122 \\
\hline & & & $\mathrm{b}$ & 91.90 & & 2.6 & & 90 & 0.04 & 0.02 & 172 \\
\hline \multirow[t]{3}{*}{ B1641+173 } & $3 \mathrm{C} 346$ & $\mathrm{G}$ & a12 & 171.30 & 0.15 & $1.2 / 14.0$ & 0.34 & -35 & 0.14 & 0.08 & 95 \\
\hline & & & a3 & 10.20 & & 1.2 & & -1 & 0.18 & 0.09 & 115 \\
\hline & & & $\mathrm{b}$ & 239.70 & & & & & 0.005 & 0.003 & 133 \\
\hline B1829+290 & 4C 29.56 & $\mathrm{G}$ & $\mathrm{a}$ & - & 0.24 & & 0.30 & & - & - & - \\
\hline & & & $\mathrm{c}$ & 356.70 & & 2.9 & & 52 & 0.06 & 0.01 & 86 \\
\hline & & & $\mathrm{c} 1$ & 2.60 & & & & & 0.20 & 0.02 & 51 \\
\hline & & & $\mathrm{d}$ & - & & & & & - & - & - \\
\hline B2247+140 & $4 C 14.82$ & Q & a & 376.30 & 0.24 & 18.5 & 0.32 & -85 & 0.07 & 0.03 & 63 \\
\hline & & & $\mathrm{b}$ & 207.40 & & 7.9 & & 13 & 0.13 & 0.06 & 162 \\
\hline
\end{tabular}

Notes. Column 1: source name; 2: other name; 3: optical identification; 4: component; 5 : integrated flux density of the components; 6: three $\sigma$ error on the total-intensity image; 7: polarised flux density; 8: three $\sigma$ error on the polarised-intensity image; 9: EVPA; 10-12: deconvolved sizes of the components; major axis, minor axis, and position angle of the major axis, respectively. 
Table 6. Polarisation parameters for the components of each source at $23285 \mathrm{MHz}$.

\begin{tabular}{|c|c|c|c|c|c|c|c|c|c|c|c|}
\hline Name & Other name & ID & Comp & $\begin{array}{c}S \\
\mathrm{mJy}\end{array}$ & $\begin{array}{l}3 \sigma \\
\mathrm{mJy} / \mathrm{b}\end{array}$ & $\begin{array}{l}S p \\
\text { mJy }\end{array}$ & $\begin{array}{l}3 \sigma p \\
\mathrm{mJy} / \mathrm{b}\end{array}$ & $\begin{array}{c}\chi \\
\operatorname{deg}\end{array}$ & $\begin{array}{l}\text { Maj } \\
\text { arcsec }\end{array}$ & $\begin{array}{l}\text { Min } \\
\text { arcsec }\end{array}$ & $\begin{array}{r}\text { PA } \\
\text { deg }\end{array}$ \\
\hline \multirow[t]{2}{*}{ B0023-263 } & & G & a12 & 489.3 & 0.42 & 3.2 & 0.70 & 43 & 0.07 & 0.01 & 152 \\
\hline & & & $\mathrm{b}$ & 316.8 & & 8.6 & & 34 & 0.06 & 0.03 & 131 \\
\hline В0114-211 & & G & & 141.3 & 0.27 & 7.1 & 0.47 & 1 & 0.054 & 0.019 & 112 \\
\hline B0116+319 & 4C 31.04 & $\mathrm{G}$ & & 550.2 & 0.27 & & 0.30 & & 0.10 & 0.026 & 112 \\
\hline \multirow[t]{3}{*}{$\mathrm{B} 0127+233$} & $3 \mathrm{C} 43$ & Q & a1 & 113.0 & 0.24 & 5.0 & 0.28 & 3 & 0.14 & 0.02 & 178 \\
\hline & & & a2 & 79.2 & & 5.6 & & 48 & 0.08 & 0.05 & 124 \\
\hline & & & $\mathrm{b}$ & 25.7 & & & & & - & - & - \\
\hline \multirow[t]{3}{*}{ B $0223+341$} & $4 C 34.07$ & Q & $\mathrm{a}$ & 610.2 & 0.21 & 5.3 & 0.36 & 1 & 0.05 & 0.005 & 114 \\
\hline & & & $\mathrm{b}$ & 12.4 & & & & & 0.126 & 0.052 & 82 \\
\hline & & & b0 & 3.1 & & & & & 0.09 & 0.09 & 51 \\
\hline B1358+624 & $4 C 62.22$ & G & & 486.0 & 0.30 & & 0.43 & & 0.03 & 0.003 & 118 \\
\hline \multirow{5}{*}{ B1416+067 } & 3C 298 & Q & $\mathrm{a}$ & 36.8 & 0.24 & 1.0 & 0.36 & -70 & 0.13 & 0.08 & 65 \\
\hline & & & $\mathrm{b}$ & 16.1 & & 0.96 & & 1 & 0.16 & 0.06 & 81 \\
\hline & & & $\mathrm{c} 1$ & 42.1 & & 3.0 & & 23 & 0.16 & 0.03 & 114 \\
\hline & & & $\mathrm{c} 2$ & 83.3 & & 0.8 & & $73 / 37$ & 0.04 & 0.008 & 118 \\
\hline & & & $\mathrm{d}$ & 18.9 & & 0.4 & & -88 & 0.09 & 0.06 & 85 \\
\hline \multirow[t]{5}{*}{ B1458+718 } & 3C 309.1 & Q & $\mathrm{a}$ & 92.1 & 0.48 & 5.4 & 0.43 & $90 /-25$ & 0.19 & 0.16 & 161 \\
\hline & & & $\mathrm{a} 0$ & & & & & & - & - & - \\
\hline & & & a1 & 26.5 & & 2.6 & & -35 & 0.023 & 0.08 & 93 \\
\hline & & & $\mathrm{b}$ & 767.6 & & 41.9 & & 46 & 0.048 & 0.020 & 151 \\
\hline & & & $\mathrm{d}$ & 11.8 & & & & & 0.23 & 0.12 & 92 \\
\hline \multirow[t]{2}{*}{ B1524-136 } & & Q & $\mathrm{a}$ & 279.0 & 0.30 & 4.9 & 0.47 & 65 & 0.10 & 0.012 & 163 \\
\hline & & & $\mathrm{b}$ & 35.3 & & 0.6 & & 88 & 0.06 & 0.05 & 74 \\
\hline \multirow[t]{2}{*}{ B1638+124 } & $4 C 12.60$ & E & $\mathrm{a}$ & 304.4 & 0.21 & 3.3 & 0.36 & 81 & 0.0 & 0.0 & 0 \\
\hline & & & $\mathrm{b}$ & 56.5 & & 2.8 & & 76 & 0.06 & 0.02 & 142 \\
\hline \multirow[t]{4}{*}{ B1641+173 } & $3 \mathrm{C} 346$ & $\mathrm{G}$ & a12 & 79.8 & 0.30 & $1.3 / 10.4$ & 0.38 & -43 & 0.12 & 0.07 & 85 \\
\hline & & & a3 & 5.1 & & 1.3 & & -1 & 0.12 & 0.08 & 163 \\
\hline & & & $\mathrm{b}$ & 234.2 & & & & & 0.003 & 0.0 & 106 \\
\hline & & & $\mathrm{c}$ & 43.6 & & & & & 1.41 & 0.82 & 95 \\
\hline B1829+290 & 4C 29.56 & $\mathrm{G}$ & $\mathrm{c}$ & 182.1 & & & & & 0.059 & 0.008 & 87 \\
\hline \multirow[t]{2}{*}{ B $2247+140$} & $4 C 14.82$ & Q & $\mathrm{a}$ & 257.5 & 0.18 & 14.8 & 0.32 & $60 /-76$ & 0.074 & 0.024 & 56 \\
\hline & & & $\mathrm{b}$ & 159.5 & & 3.8 & & 14 & 0.21 & 0.06 & 17 \\
\hline
\end{tabular}

Notes. Column 1: source name; 2: other name; 3: optical identification; 4: component; 5: flux density; 6: three $\sigma$ error on the total-intensity image; 7: polarised flux density; 8: three $\sigma$ error on the polarised-intensity image; 9: EVPA; 10-12: deconvolved sizes of the components; major axis, minor axis, and position angle of the major axis, respectively.

polarised emission, and is almost certainly the core of the source. Both lobes are polarised at $X$ - and $U$-band, showing strong depolarisation between 15 and $8.0 \mathrm{GHz}$. The lobes are completely depolarised by $5 \mathrm{GHz}$ as reported by Lüdke et al. (1998). The eastern and western lobes have RMs of 942 and $615 \mathrm{rad} \mathrm{m}^{-2}$ respectively.

3C 237 has also been observed by Akujor \& Garrington (1995) at $8.4 \mathrm{GHz}$ and by van Breugel et al. (1992) at $15 \mathrm{GHz}$.

1151-348 - This is a point-like source at sub-arcsecond resolution. It is up to $2.1 \%$ polarised at $15 \mathrm{GHz}$ and shows depolarisation towards $X$-band, with an RM of about zero. Dual-band $\mathrm{S} / \mathrm{X}$ VLBI observations made by Tzioumis et al. (2002) revealed that 1151-348 has a double structure with the components separated by about 90 milli-arcseconds.

$1153+317$ (4C 31.38) - Our observations at $8 \mathrm{GHz}$ show this to be a double source, with both components being polarised. From the observations of Lonsdale et al. (1993), it is possibly a triple source at $15 \mathrm{GHz}$. Lonsdale et al. (1993) also observed the source at $5 \mathrm{GHz}$. Adopting their polarisation measurements, we compute RMs of 913 and $351 \mathrm{rad} \mathrm{m}^{-2}$ for components $a$ and $b$ respectively.

$1210+134(4 C 13.46)$ - This source presents a very complex structure, dominated by the northern component in which two blobs of emission, both strongly polarised, are embedded in an extended region of weak emission (Fig. 1). A three-arcsecond long jet extends south, beginning from the south-western most of the two blobs mentioned above. Polarised emission is detected all along the jet at $X$-band. The south-western and north-eastern blobs show $\mathrm{RM}=640$ and $-160 \mathrm{rad} \mathrm{m}^{-2}$ respectively. The values we find for the percentage polarisations are rather puzzling: the brightest component $a$ depolarises going from 15 to $8.4 \mathrm{GHz}$ $\left(D P_{8485 / 14885}=0.7\right)$, while component $b$ strongly repolarises $\left(D P_{8485 / 14885}=2.3\right)$. Polarised emission is also detected at the three observing frequencies for component $f$ at the end of the jet, which also shows repolarisation and has $\mathrm{RM}=1597 \mathrm{rad} \mathrm{m}^{-2}$.

1245-197 - This source is slightly resolved at $15 \mathrm{GHz}$. The image shows an extension to the west of the brightest point-like component. This component is weakly polarised, and has a very large depolarisation index. It is one of the few cases that does not follow the $\lambda^{2}$ law. 1245-197 was also observed with the VLA at $1.36 \mathrm{GHz}$ by Stanghellini et al. (2005) who found a component about 4 arcmin to the west of the main component.

$1311+678,4 C 67.22$ - Polarised emission is not detected for this source which is slightly extended to the south-east at $15 \mathrm{GHz}$. $1311+678$ shows double structure at milli-arcsecond resolution (see $\mathrm{Xu}$ et al. 1995).

$1323+321$ (4C 32.44) - Again a point-like source, it is slightly depolarised between $15 \mathrm{GHz}$ and $X$-band. However, 
F. Mantovani et al.: Radio polarimetry of compact steep spectrum sources at sub-arcsecond resolution

Table 7. Fractional polarisation, depolarisation index and rotation measure for individual components of each source.

\begin{tabular}{|c|c|c|c|c|c|c|c|c|c|c|c|c|}
\hline Name & Other name & ID & Comp & $\begin{array}{l}m_{8.0} \\
{[\%]}\end{array}$ & $\begin{array}{l}m_{8.4} \\
{[\%]}\end{array}$ & $\begin{array}{l}m_{14.8} \\
{[\%]}\end{array}$ & $\begin{array}{l}m_{23.2} \\
{[\%]}\end{array}$ & $D P_{8.0 / 8.4}$ & $D P_{8.4 / 14.8}$ & $D P_{14.8 / 23.2}$ & 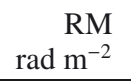 & $\begin{array}{r}\mathrm{RM}_{\mathrm{rf}} \\
\mathrm{rad} \mathrm{m}^{-2}\end{array}$ \\
\hline B0023-263 & & G & $\begin{array}{l}\mathrm{a} \\
\mathrm{b}\end{array}$ & $\begin{array}{l}0.43 \\
0.39\end{array}$ & $\begin{array}{l}0.30 \\
0.22\end{array}$ & $\begin{array}{l}0.67 \\
0.67\end{array}$ & $\begin{array}{l}0.65 \\
2.71\end{array}$ & $\begin{array}{l}1.43 \\
1.64\end{array}$ & $\begin{array}{l}0.45 \\
0.33\end{array}$ & $\begin{array}{l}1.03 \\
0.25\end{array}$ & 146 & 255 \\
\hline $\begin{array}{l}\text { B0114-211 } \\
\text { B0116+319 }\end{array}$ & $4 C 31.04$ & $\begin{array}{l}\mathrm{G} \\
\mathrm{G}\end{array}$ & & 0.27 & 0.18 & & 5.02 & 1.50 & & & 646 & 3752 \\
\hline B $0127+233$ & $3 \mathrm{C} 43$ & Q & $\begin{array}{l}\text { a1 } \\
\text { a2 } \\
\text { b } \\
\text { c }\end{array}$ & $\begin{array}{l}9.34 \\
5.69\end{array}$ & $\begin{array}{l}12.09 \\
7.45\end{array}$ & $\begin{array}{l}4.71 \\
7.22\end{array}$ & $\begin{array}{l}4.1 \\
7.07\end{array}$ & $\begin{array}{l}1.03 \\
\\
0.72 \\
0.76\end{array}$ & 0.93 & $\begin{array}{l}1.67 \\
1.02\end{array}$ & $\begin{array}{l}-24 \\
-23\end{array}$ & $\begin{array}{l}-145 \\
-139\end{array}$ \\
\hline B0221+276 & 3C 67 & G & $\begin{array}{l}\text { a1 } \\
\text { a2 } \\
\text { b }\end{array}$ & 8.57 & 8.13 & $\begin{array}{l}5.36 \\
2.02\end{array}$ & & 1.05 & 1.51 & & $\begin{array}{r}768 \\
942 \\
1745\end{array}$ & $\begin{array}{l}1318 \\
1617 \\
2995\end{array}$ \\
\hline B0223+341 & 4C 34.07 & Q & $\begin{array}{l}\mathrm{a} \\
\mathrm{b}\end{array}$ & $\begin{array}{l}0.37 \\
1.66\end{array}$ & $\begin{array}{l}0.29 \\
1.45\end{array}$ & $\begin{array}{l}0.38 \\
7.39\end{array}$ & 0.87 & $\begin{array}{l}1.28 \\
1.14\end{array}$ & $\begin{array}{l}0.76 \\
0.20\end{array}$ & 0.44 & $\begin{array}{r}-2373 \\
384\end{array}$ & $\begin{array}{r}-36279 \\
5871\end{array}$ \\
\hline B0319+121 & & & & 4.40 & 4.20 & 4.66 & & 1.05 & 0.90 & & 0 & 0 \\
\hline $\begin{array}{l}\text { B } 0404+767 \\
\text { B0531+194 }\end{array}$ & 4C 76.03 & $\begin{array}{l}E \\
G\end{array}$ & $\begin{array}{l}\mathrm{a} \\
\mathrm{b}\end{array}$ & 0.20 & $\begin{array}{l}0.06 \\
0.13 \\
0.06\end{array}$ & 3.66 & & 3.33 & 0.02 & & 2090 & 5350 \\
\hline B $0538+498$ & $3 \mathrm{C} 147$ & Q & a1 & 0.72 & 0.74 & $\begin{array}{l}4.12 \\
0.28 \\
8.48\end{array}$ & & 0.97 & 0.18 & & & \\
\hline B $1005+077$ & 3C 237 & G & $\mathrm{a}$ & $\begin{array}{l}0.95 \\
0.15\end{array}$ & $\begin{array}{l}1.05 \\
0.25\end{array}$ & $\begin{array}{l}0.40 \\
4.73 \\
1.86\end{array}$ & & $\begin{array}{l}0.01 \\
0.90 \\
0.60\end{array}$ & $\begin{array}{l}0.22 \\
0.13\end{array}$ & & $\begin{array}{l}-174 \\
-942\end{array}$ & $\begin{array}{r}-615 \\
-3329\end{array}$ \\
\hline B1151-348 & & Q & & 1.3 & 1.25 & 2.1 & & 1.04 & 0.60 & & 0 & 0 \\
\hline B1153+317 & 4C 31.38 & Q & $\begin{array}{l}\mathrm{a} \\
\mathrm{b}\end{array}$ & $\begin{array}{l}0.85 \\
4.51\end{array}$ & $\begin{array}{l}0.68 \\
4.72\end{array}$ & & & $\begin{array}{l}1.25 \\
0.96\end{array}$ & & & $\begin{array}{l}453 \\
174\end{array}$ & $\begin{array}{l}913 \\
351\end{array}$ \\
\hline B1210+134 & 4C 13.46 & Q & $\begin{array}{l}a \\
b \\
d \\
e\end{array}$ & $\begin{array}{r}7.15 \\
4.20 \\
20.45 \\
3.08\end{array}$ & $\begin{array}{l}7.00 \\
4.60 \\
20.79 \\
7.69\end{array}$ & $\begin{array}{l}9.82 \\
1.98\end{array}$ & & $\begin{array}{l}1.02 \\
0.91 \\
0.98 \\
0.40\end{array}$ & $\begin{array}{l}0.71 \\
2.32\end{array}$ & & $\begin{array}{l}140 \\
-35\end{array}$ & $\begin{array}{r}640 \\
-160\end{array}$ \\
\hline & & & $\mathrm{f}$ & 21.57 & 25.17 & 17.0 & & 0.86 & 1.48 & & 349 & 1597 \\
\hline B1245-197 & & Q & a & 0.07 & 0.15 & 0.62 & & 0.47 & 0.24 & & & \\
\hline B1323+321 & 4C 32.44 & G & & 0.78 & 0.93 & 1.10 & & 0.84 & 0.85 & & -35 & $\begin{array}{r}-66 \\
556\end{array}$ \\
\hline $\begin{array}{l}\text { B1328+254 } \\
\text { B1345+125 }\end{array}$ & $\begin{array}{l}3 \mathrm{C} 287 \\
4 \mathrm{C} 12.50\end{array}$ & Q & & $\begin{array}{l}4.15 \\
0.04\end{array}$ & $\begin{array}{l}3.94 \\
0.15\end{array}$ & $\begin{array}{l}4.23 \\
0.58\end{array}$ & & $\begin{array}{l}1.05 \\
0.27\end{array}$ & 0.93 & & $\begin{array}{r}-131 \\
-5585\end{array}$ & $\begin{array}{r}-556 \\
-8271\end{array}$ \\
\hline B1358+624 & 4C 62.22 & G & & 0.38 & 0.24 & 0.39 & & $\begin{array}{l}0.21 \\
1.58\end{array}$ & 0.61 & & -3665 & -7496 \\
\hline B1416+067 & 3C 298 & Q & $\begin{array}{l}\mathrm{a} \\
\mathrm{b} \\
\mathrm{c} 1 \\
\mathrm{c} 2 \\
\mathrm{~d}\end{array}$ & 7.60 & 7.59 & $\begin{array}{l}7.14 \\
13.17 \\
0.99\end{array}$ & $\begin{array}{l}2.72 \\
5.96 \\
7.13 \\
0.96 \\
2.12\end{array}$ & 1.00 & 1.06 & $\begin{array}{l}2.63 \\
2.21 \\
0.14\end{array}$ & $\begin{array}{l}-140 \\
-175\end{array}$ & $\begin{array}{r}-833 \\
-1042\end{array}$ \\
\hline B1458+718 & 3C 309.1 & Q & $\begin{array}{c}\mathrm{a} \\
\mathrm{a} 1 \\
\mathrm{~b} \\
\mathrm{c}\end{array}$ & $\begin{array}{l}7.56 \\
\\
5.02 \\
2.27\end{array}$ & 7.33 & $\begin{array}{l}10.05 \\
8.62 \\
5.53\end{array}$ & $\begin{array}{l}5.86 \\
9.81 \\
5.46\end{array}$ & 1.03 & $\begin{array}{l}0.73 \\
0.91\end{array}$ & $\begin{array}{l}1.71 \\
0.88 \\
1.01\end{array}$ & $\begin{array}{r}-1047 \\
314\end{array}$ & $\begin{array}{r}-3920 \\
1146\end{array}$ \\
\hline & & & $\begin{array}{l}\mathrm{d} \\
\mathrm{e}\end{array}$ & $\begin{array}{l}1.80 \\
2.49\end{array}$ & 2.11 & 1.8 & & 0.85 & & & 2792 & 10185 \\
\hline B1524-136 & OQ 172 & Q & $\begin{array}{l}a \\
b\end{array}$ & & & & $\begin{array}{l}1.76 \\
1.70\end{array}$ & & & & 2827 & 20411 \\
\hline B1634+628 & 3 C 343 & G & $\mathrm{a}$ & 1.20 & 1.17 & 1.36 & & 1.03 & 0.86 & & 698 & 2764 \\
\hline B $1638+124$ & 4C 12.60 & E & $\begin{array}{l}\mathrm{a} \\
\mathrm{b}\end{array}$ & 0.22 & 0.21 & $\begin{array}{l}0.16 \\
2.83\end{array}$ & $\begin{array}{l}1.08 \\
4.96\end{array}$ & 1.05 & 1.31 & $\begin{array}{l}0.15 \\
0.57\end{array}$ & 3141 & 14546 \\
\hline B1641+173 & $3 C 346$ & G & $\begin{array}{c}\text { a12 } \\
\text { a3 }\end{array}$ & 7.72 & 7.41 & $\begin{array}{l}8.87 \\
11.76\end{array}$ & $\begin{array}{l}14.66 \\
25.49\end{array}$ & 1.04 & 0.84 & $\begin{array}{l}0.60 \\
0.46\end{array}$ & $\begin{array}{l}54 \\
17\end{array}$ & $\begin{array}{l}73 \\
23\end{array}$ \\
\hline B1829+290 & 4C 29.56 & G & $\begin{array}{l}\mathrm{c} \\
\mathrm{e}\end{array}$ & $\begin{array}{l}0.61 \\
2.91\end{array}$ & $\begin{array}{l}0.49 \\
1.82\end{array}$ & 0.81 & & $\begin{array}{l}1.24 \\
1.60\end{array}$ & 0.60 & & -1466 & -4963 \\
\hline B2247+140 & 4C14.82 & Q & $\begin{array}{l}\mathrm{a} \\
\mathrm{b}\end{array}$ & 2.90 & 3.02 & $\begin{array}{l}4.92 \\
3.81\end{array}$ & $\begin{array}{l}5.75 \\
2.38\end{array}$ & 0.96 & 0.61 & $\begin{array}{l}0.85 \\
1.60\end{array}$ & $\begin{array}{l}209 \\
454\end{array}$ & $\begin{array}{l}319 \\
692\end{array}$ \\
\hline
\end{tabular}

Notes. Column 1: source name; 2: other name; 3: optical identification; 4: component; 5: percentage of polarised emission at 8085 MHz; 6: percentage of polarised emission at $8485 \mathrm{MHz}$; : percentage of polarised emission at $14885 \mathrm{MHz}$; 8: percentage of polarised emission at $23285 \mathrm{MHz}$; depolarisation index between $8485 \mathrm{MHz}$ and $8085 \mathrm{MHz}$; 10: depolarisation index between $14885 \mathrm{MHz}$ and $8485 \mathrm{MHz}$; 11: depolarisation index between $23285 \mathrm{MHz}$ and $14885 \mathrm{MHz}$; 12: rotation measure; 13: rotation measure in the source rest frame $\mathrm{RM}_{\mathrm{rf}}=\mathrm{RM} \times(1+z)^{2}$. 
the percentage polarisation is low. The value of the intrinsic $\mathrm{RM}$ is also low $\left(\mathrm{RM}_{\mathrm{rf}}=-66 \mathrm{radm}^{-2}\right) .1323+321$ is part of the MOJAVE monitoring programme which is performed with the VLBA at $15 \mathrm{GHz}$. There it shows a double structure (Lister \& Homan 2005) with the two components being separated by about 50 milli-arcsec.

$1328+254$ (3C 287) - This point-like source is about $4 \%$ polarised, with little depolarisation from higher to lower frequencies. The intrinsic $\mathrm{RM}_{\mathrm{rf}}$ is $-556 \mathrm{rad} \mathrm{m}^{-2}$. 3C 287 was observed by Kellermann et al. (1998) with the VLBA at $15 \mathrm{GHz}$ and showed a point-like structure with a weak extension to the south. $1345+125$ (4C 12.50) - A point-like source that is only weakly polarised. It presents a very high depolarisation index going from higher to lower frequency and a very large intrinsic RM $\left(\mathrm{RM}_{\mathrm{rf}}=-8271 \mathrm{rad} \mathrm{m}^{-2}\right)$. VLBI observations by Stanghellini et al. (1997) at $5 \mathrm{GHz}$ and Kellermann et al. (1998) at $15 \mathrm{GHz}$ reveal a complex, long, thin structure for the source.

$1358+624$, (4C 62.22) - The present observations show a pointlike structure for $1358+624$. It is weakly polarised, and presents noticeable depolarisation, with a large intrinsic $\mathrm{RM}\left(\mathrm{RM}_{\mathrm{rf}}=\right.$ $-7496 \mathrm{rad} \mathrm{m}^{-2}$ ). VLBI observations at $18 \mathrm{~cm}$ by Dallacasa et al. (1995) show a double structure with a faint bridge, identified as a jet, connecting the two lobes.

$1416+067$, (3C 298) - This source extends east-west, and is resolved into multiple components. We can identify at least five of these in our $23.2 \mathrm{GHz}$ image, which shows percentage polarisations ranging from about $1 \%$ to $7 \%$ at $23.2 \mathrm{GHz}$. Polarised emission was detected only on the eastern side at $X$-band, while three components show polarisation at $15 \mathrm{GHz}$. Unexpectedly, components $a$ and $b$ show repolarisation having $D P_{14885 / 23285}=2.6$ and 2.2 respectively, while component $c 1$ is strongly depolarised $\left(D P_{14885 / 23285}=0.14\right)$. Component $a$ is the only one found to be polarised at $X$-band. It shows depolarisation going from 15 to $8.0 \mathrm{GHz}$.

$1416+067$ has been the target of many sub- and milliarcsecond resolution observations. It has been observed by Akujor \& Garrington (1995) at 1.6 and $8.4 \mathrm{GHz}$ with the VLA, Lüdke et al. (1998) with MERLIN at $5.0 \mathrm{GHz}$, and by van Breugel et al. (1992) at $15 \mathrm{GHz}$ with the VLA. We incorporated the values of $\chi$ measured for some of the components by these observers to better calculate the RMs. Components $a$ and $b$ show $\mathrm{RM}_{\mathrm{rf}}=-883$ and $-310 \mathrm{rad} \mathrm{m}^{-2}$, respectively. However, we obtained a much better fit to the $\lambda^{2}$ law for component $b$ $\left(\mathrm{RM}_{\mathrm{rf}}=-1042 \mathrm{rad} \mathrm{m}^{-2}\right)$ if the $\chi$ value at $5.0 \mathrm{GHz}$ of Lüdke et al. (1998) is discarded.

The structure of 3C 298 at milli-arcsecond resolution is discussed in depth by Fanti et al. (2002) and references therein. They observed the source with the EVN at wavelengths of 92 , 18 and $6 \mathrm{~cm}$.

$1458+718$ (3C 309.1) - The sub-arcsecond structure of $1458+718$ is characterized by several components. Three of these are aligned in the east-west direction, while the others lie along the extended emission pointing south, which is clearly detected in both of our $X$-band images. Polarised emission is detected for the two brightest components, $a$ and $b$, at all four of our observing frequencies

3C 309.1 has been the target of many other investigations. We mention MERLIN observations at $5 \mathrm{GHz}$ by Lüdke et al. (1998), and by Akujor \& Garrington (1995) at 1.4, 5, 8.4, and $15 \mathrm{GHz}$. The $\chi$ values reported by Lüdke et al. (1998), obtained from observations made at comparable resolution, were added to our measurements in order to derive the RMs of three of the components: $\mathrm{RM}_{\mathrm{rf}}=-3920 \mathrm{rad} \mathrm{m}^{-2}$ for component $a$ $\mathrm{RM}_{\mathrm{rf}}=1146 \mathrm{rad} \mathrm{m}^{-2}$ for $a 1$, and $\mathrm{RM}_{\mathrm{rf}}=10185 \mathrm{rad} \mathrm{m}^{-2}$ for $d$.
VLBI images for 3C 309.1 can be found in Xu et al. (1995) at $1.6 \mathrm{GHz}$, and in Kellermann et al. (1998) and Lister \& Homan (2005) at $15 \mathrm{GHz}$. We note that the milli-arcsec images show a north-south core-jet structure, while the source major axis in images at lower resolution is east-west.

$1524-136(O Q 172)$ - We only observed this source at $23.2 \mathrm{GHz}$ since it had already been observed by Mantovani et al. (1994) with the VLA at 6,4 , and $2 \mathrm{~cm}$. It has a double structure, and polarised emission is detected for both of the components at $23.2 \mathrm{GHz}$. At lower frequencies the polarisation is below the detection limits for the weaker southern component. Combining the observations mentioned here we found the very large $\mathrm{RM}_{\mathrm{rf}}$ of $20411 \mathrm{rad} \mathrm{m}^{-2}$ for the stronger northern component, with virtually no depolarisation from 23.2 to $5.0 \mathrm{GHz}$.

OQ172 was observed in VLBI by Udomprasert et al. (1997), who find a component close to the source nucleus with an $\mathrm{RM}_{\mathrm{rf}}$ up to $40000 \mathrm{rad} \mathrm{m}^{-2}$, and by Mantovani et al. (2002), who pointed out that the source is a quasar with a two-sided jet, which is unusual for this class of objects.

$1634+628$, (3C 343) - This source shows a core-jet structure in the VLA images at $X$-band. The jet (or extension) is not detected at $15 \mathrm{GHz}$. Polarised emission is detected for the brightest component, showing very little depolarisation and a $\mathrm{RM}_{\mathrm{rf}}=$ $2764 \mathrm{rad} \mathrm{m}^{-2}$. Polarimetric VLBA observations at 5 and $8.4 \mathrm{GHz}$ for 3C 343 were recently published by Mantovani et al. (2010). Two bright, compact components, surrounded by weak, diffuse emission are detected; both components are polarised. The whole polarised region depolarises little. However, the $\chi$ values present large changes, with the $\mathrm{RM}_{\mathrm{rf}}$ varying across the source, with indications of values higher than $6000 \mathrm{rad} \mathrm{m}^{-2}$.

$1638+124$ (4C 12.60) - The source $1638+124$ shows a double structure at the higher of our observing frequencies, appearing slightly resolved at $15 \mathrm{GHz}$, but point-like at $X$-band. A value for $\mathrm{RM}_{\mathrm{rf}}=14546 \mathrm{rad} \mathrm{m}^{-2}$ is derived for component $a$, which also shows $D P_{14885 / 23285}=0.15$.

$1641+173(3 C 346)$ - The structure of $3 C 346$ is that of an asymmetric triple source. The western lobe is completely resolved out at 15 and $23.2 \mathrm{GHz}$. Strong polarised emission (up to $17 \%$ ) is found for the brightest component of the eastern lobe. This component depolarises quickly with $D P_{14885 / 23285}=0.51$, while the $\mathrm{RM}_{\mathrm{rf}}$ is below $73 \mathrm{rad} \mathrm{m}^{-2}$. This source has also been observed at $1.6,5$, and $8.4 \mathrm{GHz}$ by Akujor \& Garrington (1995) at sub-arcsecond resolution, and by Spencer et al. (1991) with MERLIN and EVN at $18 \mathrm{~cm}$, and Cotton et al. (1995) at 1.7 and $8.4 \mathrm{GHz}$

$1829+290$ (4C 29.56) - This source possesses a triple structure. The central component is the brightest and is polarised at both $X$-band and $15 \mathrm{GHz}$, while polarised emission is not detected at 23.2 GHz. Weak polarised emission is also detected for the western lobe at $X$-band. The two external lobes and the two-sided jet are below the detection limits at the two higher frequencies. The derived $\mathrm{RM}_{\mathrm{rf}}$ of $4963 \mathrm{rad} \mathrm{m}^{-2}$ is questionable.

$1829+290$ was observed with the EVN at $1.6 \mathrm{GHz}$ by Dallacasa et al. (1995). Their image shows symmetric, bright, elongated features, likely to be jets, at a position angle of about $90^{\circ}$. This is strongly misaligned with respect to the outer lobes seen on the sub-arcsecond resolution images. The radio core is not detected by Dallacasa et al.

$2247+140$ (4C 14.82) - The $23.2 \mathrm{GHz}$ image of this source shows it to have a double structure. At the lower resolution of $X$-band, it appears to be point-like, while the double structure can be recognised at $15 \mathrm{GHz}$. Although, the structure of the polarised emission allowed us to easily separate the two regions, even at the lower resolution, it is difficult to derive the percentage 
polarisations. It is easier to derive the $\chi$ values for the two components, and this has allowed us to calculate $\mathrm{RM}_{\mathrm{rf}}=319$ and $692 \mathrm{rad} \mathrm{m}^{-2}$.

\section{Discussion}

\subsection{Fractional polarisation}

Considering the values of the fractional polarisation listed in Table 1, our VLA A-array observations confirm that CSSs are weakly polarised. However, we find that quasars are more highly polarised than galaxies, and in both cases the polarisation percentages, $m$, decrease from higher to lower frequencies. This is consistent with a number of earlier results (see e.g. Saikia et al. 1985, 1987; Saikia \& Salter 1988; Akujor \& Garrington 1995; Cotton et al. 2003a). The differences between galaxies and quasars could be due to a combination of orientation effects and the relative contribution of the jet, which is often more strongly polarised. Point-like sources are the least polarised with a median value of $m \simeq 1 \%$. The median $\mathrm{m}$ found at $X$-band confirms the finding by Mantovani et al. (2009) that the median value of $m$ for a complete sample of CSSs observed at the same band was lower than $1 \%$.

The median value of $\mathrm{m}$ for individual components in both classes of optical objects decreases slightly going from higher to lower frequencies. However, considering galaxies and quasars separately, we note that the above is confirmed for quasars down to $8085 \mathrm{MHz}$, while galaxies, in contrast, show strong depolarisation between 14885 and $8085 \mathrm{MHz}$.

A comparison can be made with the sample of steep spectrum extended radio sources selected from the B3-VLA sample observed at $4.85 \mathrm{GHz}$ by Klein et al. (2003). These authors found a fractional polarisation of $5.2 \%$.

\subsection{Rotation measures}

RMs have been calculated for point sources and for many individual components of the sources that are resolved by the VLA A-array. The median values for the modulus $\left|\mathrm{RM}_{\mathrm{rf}}\right|$ are $1146_{-531}^{+2183} \mathrm{radm}^{-2}$ for the whole set of source components, $692_{-532}^{+887} \mathrm{rad} \mathrm{m}^{-2}$ for components in quasars, and $1617_{-1298}^{+2135} \mathrm{rad} \mathrm{m}^{-2}$ for components in galaxies. We have 10 galaxies and 6 quasars containing components with RMs higher than the $|\mathrm{RM}|$, while 4 galaxies and 13 quasars have RMs lower than this. The dispersion of the RMs is large, ranging from $0 \mathrm{rad} \mathrm{m}^{-2}$ to about $36000 \mathrm{rad} \mathrm{m}^{-2}$ in the source rest frame.

A narrower dispersion was found for a sample of 47 CSSs observed with the Effelsberg 100-m telescope by Mantovani et al. (2009). RMs for the 16 sources with polarised emission greater than the detection limits lie between -20 and $3900 \mathrm{rad} \mathrm{m}^{-2}$. This might be expected since sources that are unresolved by single dish observations should show lower levels of polarisation (and RMs) due to the blending of emission from multiple components having different EVPAs. However, among the 12 point-like sources of the present sample, half have RMs close to zero, while 6 show RMs $>500 \mathrm{rad} \mathrm{m}^{-2}$, of which 3 are classified as galaxies.

For comparison, for a sample of faint blazars observed with the Effelsberg 100-m telescope it is found that the RMs range between 0 and $1950 \mathrm{radm}^{-2}$. Only 9 out of the 27 objects with polarised emission above the detection limits show RM > $200 \mathrm{rad} \mathrm{m}^{-2}$ (Mantovani et al. (2011).
The lower values of RM for the components in the galaxies compared with quasars is interesting. In the unified scheme this could arise if the quasar components are seen largely through the less dense ionization cones, while the galaxy components may be seen through the torus or the denser interstellar medium of the host galaxy. The errors on the median values are rather large now, and it would be useful to extend such studies to a larger sample of sources. Also although single-dish values have yielded lower values of RM, it is important to study these sources with high resolution to determine their polarimetric properties. A deeper study of the results achieved by this investigation will be given in a future paper.

\section{Summary and conclusions}

We have presented multi-frequency VLA polarisation observations of CSSs and estimated their percentage polarisations and RM values.

About half of the sources are point-like, even at the $\sim 0.1 \times$ 0.1 arcsec resolution achieved by the present VLA A-array 23.2-GHz observations. The remaining sources have double or triple structures. One source, $1210+134$ (4C 13.46) shows a complex structure.

Low values for the polarisation percentage in CSSs is confirmed by the present observations. On the average, quasars are more highly polarised than galaxies.

We have compiled the available RM estimates for CSS sources as seen on a sub-arcsecond scale. These show a wide range of values, with indications of very large RMs, although some of the values need to be confirmed via observations at a larger number of frequencies. Values of $\mathrm{RM}_{\mathrm{rf}}$ as high as $\approx 36000 \mathrm{rad} \mathrm{m}^{-2}$ have been found. CSS galaxies are characterized by RM values that are larger than those found for CSS quasars. A majority of the objects show very large values of $\mathrm{RM}_{\mathrm{rf}}$.

Acknowledgements. The VLA is operated by the U.S. National Radio Astronomy Observatory, which is a facility of the National Science Foundation operated under a cooperative agreement by Associated Universities, Inc. This research has made use of data from the MOJAVE database that is maintained by the MOJAVE team (Lister et al. 2009). It has also used the NASA/IPAC Extragalactic Database (NED) which is operated by the Jet Propulsion Laboratory, California Institute of Technology, under contract with the National Aeronautics and Space Administration, and NASA's Astrophysics Data System. We regret that it took so long to make these data public. We are very grateful to the referee, Prof. Ralf Spencer, for the very helpful comments and suggestions he made, and for a careful reading of the manuscript of this paper.

\section{References}

Akujor, C. E., \& Garrington, S. T. 1995, A\&AS, 112, 235

Cotton, W. D., Feretti, L., Giovannini, G., et al. 1995, ApJ, 452, 605

Cotton, W. D., Dallacasa, D., Fanti, C., et al. 2003a, A\&A, 406, 43

Cotton, W. D., Spencer, R. E., Saikia, D. J., \& Garrington, S. 2003b, A\&A, 403, 537

Dallacasa D., \& Stanghellini C. 1990, Proc. workshop Compact Steep-Spectrum \& GHz-Peaked Spectrum Radio Sources, eds. C. Fanti, R. Fanti, C. P. O’Dea, \& R. T. Schilizzi

Dallacasa, D., Fanti, C., Fanti, R., et al. 1995, A\&A, 295, 27

Fanti, R., Fanti, C., Schilizzi, R. T., et al. 1990, A\&A, 231, 333

Fanti, C., Fanti, R., Dallacasa, D., et al. 2002, A\&A, 396, 801

Junor, W., Salter, C. J., Saikia, D. J., Mantovani, F., \& Peck, A. B. 1999, MNRAS, 308, 955

Klein, U., Mack, K.-H., Gregorini, L., \& Vigotti, M. 2003, A\&A, 406, 579

Kellermann, K. I., Vermeulen, R. C., Zensus, J. A., \& Cohen, M. H. 1998, AJ, 115,1295 
A\&A 555, A4 (2013)

Lister, M. L., \& Homan, D. C. 2005, AJ, 130, 1389

Lister, M. L., Aller, H. D., Aller, M. F., et al. 2009, AJ, 137, 3718

Lonsdale, C. J., Barthel, P. D., \& Miley, G. K. 1993, ApJS, 87, 63

Lüdke, E., Garrington, S. T., Spencer, R. E., et al. 1998, MNRAS, 299, 467

Mantovani, F., Junor, W., Fanti, R. et al. 1994, A\&A, 292, 59

Mantovani, F., Junor, W., Ricci, R., et al. 2002, A\&A, 389, 58

Mantovani, F., Junor, W., Saikia, D. J., \& Salter, C. 2003, PASA, 20, 123

Mantovani, F., Mack, K.-H., Montenegro-Montes, F. M., et al. 2009, A\&A, 502,

61

Mantovani, F., Rossetti, A., Junor, W., et al. 2010, A\&A, 518, A33

Mantovani, F., Bondi, M., \& Mack, K.-H. 2011, A\&A, 533, A79

McCarthy, P. J., Kapahi, V. K., van Breugel, W., et al. 1996, ApJS, 107, 19

Nan, R. D., Schilizzi, R. T., Fanti, C., \& Fanti, R. 1991, A\&A, 252, 513
Saikia, D. J., \& Salter, C. J. 1988, Ann. Rev. Astron. Astrophys. 26, 93 Saikia, D. J., Swarup, G., \& Kodali, P. D. 1985, MNRAS, 216, 385 Saikia, D. J., Singal, A. K., \& Cornwell, T. J. 1987, MNRAS, 224, 379

Sanghera, H. S., Saikia, D. J., Lüdke, E., et al. 1995, A\&A, 295, 629 Spencer, R. E., Schilizzi, R. T., Fanti, C., et al. 1991, MNRAS, 250, 225 Stanghellini, C., O’Dea, C. P., Baum, et al. 1997, A\&A, 325, 943

Stanghellini, C., O’Dea, C. P., Dallacasa, D., et al. 2005, A\&A, 443, 891

Tzioumis, A., King, E., Morganti, R., et al. 2002, A\&A, 392, 841

Udomprasert, P. S., Taylor, G. B., Pearson, T. J., \& Roberts, D. H. 1997, ApJ, 483, L9

van Breugel, W., Miley, G., \& Heckman, T. 1984, AJ, 89, 5

van Breugel, W. J. M., Fanti, C., Fanti, R., et al. 1992, A\&A, 256, 56

Xu, W., Readhead, A. C. S., Pearson, T. J., et al. 1995, ApJS, 99, 297 